\title{
Enhanced recovery after surgery: Neuroanaesthetic perspective
}

\author{
Rajeeb K. Mishra, Indu Kapoor, Charu Mahajan, Hemanshu Prabhakar
}

\begin{abstract}
Enhanced recovery after surgery (ERAS) is a systematic multimodal perioperative care aimed at reducing the immense surgical stress of the patient and thereby facilitating early recovery. This is basically a multidiscipline, multimodal integrated approach in patient care and it integrates the basic knowledge in a more streamlined fashion, which ultimately improves the outcome of surgery. This article reviews the various aspects of ERAS, and its implementation in neurosurgical practice as some concepts of ERAS may not be applicable in the setting of neurosurgery.
\end{abstract}

Key words: Craniotomy, enhanced recovery after surgery, neuroanaesthesia, neurosurgery, spine

\section{INTRODUCTION}

Enhanced recovery after surgery (ERAS) ${ }^{[1-5]}$ is a systematic multimodal perioperative care aimed at reducing the immense surgical stress of the patient and thereby facilitating early recovery. This is basically a multidiscipline, multimodal, integrated approach in patient care and it integrates the basic knowledge in a more streamlined fashion, which ultimately improves the outcome of surgery. In other words, it is a fast tracking of perioperative processes, which leads to a better outcome. Initiated by Professor Kehlet in $1997,{ }^{[6]}$ ERAS has been applied successfully in colorectal surgery, ${ }^{[1]}$ gynaecological surgery, ${ }^{[2]}$ rectal and pelvic surgery, ${ }^{[3]}$ vascular surgery and more recently urological surgery, ${ }^{[4]}$ with good patient outcomes. Compared to traditional

Department of Neuroanaesthesiology and Critical Care, All India Institute of Medical Sciences, New Delhi, India

Address for correspondence:

Dr. Hemanshu Prabhakar, Department of Neuroanaesthesiology and Critical Care, Neurosciences Center, All India Institute of Medical

Sciences, New Delhi, India.

E-mail: prabhakaraiims@yahoo.co.in

\begin{tabular}{|l|l|}
\hline \multicolumn{2}{|c|}{ Access this article online } \\
\hline Quick Response Code: & Website: \\
\hline & www.jnaccjournal.org \\
\cline { 2 - 2 } & \\
\hline
\end{tabular}

perioperative care, adherence to ERAS protocol has been linked to better patient outcome, lesser post-operative stay and expedite recovery.

The application of ERAS in neurosurgical practice is a relatively new concept. The implementation of ERAS in craniotomies ${ }^{[7]}$ could have a significant impact in perioperative care. In neurosurgery, because of the inherent morbidity and mortality attributed to the disease per se, the outcome of the patient post-surgery degrades exponentially, if the perioperative care is not proper. Hence, there is a need for a delicate, well-balanced protocol to mark a significant difference in patient outcome. As such, there is paucity in literature regarding the implementation of a stringent protocol such as ERAS in neurosurgery.

This article reviews the various aspects of ERAS and its implementation in neurosurgical practice. In this paper, we will review various ERAS components applicable to craniotomies and major spine surgery and reconnoitre unique concepts related to enhanced recovery for neurosurgery. The protocol basically modifies the

This is an open access article distributed under the terms of the Creative Commons Attribution-NonCommercial-ShareAlike 3.0 License, which allows others to remix, tweak, and build upon the work non-commercially, as long as the author is credited and the new creations are licensed under the identical terms.

For reprints contact: reprints@medknow.com

How to cite this article: Mishra RK, Kapoor I, Mahajan C, Prabhakar $\mathrm{H}$. Enhanced recovery after surgery: Neuroanaesthetic perspective. J Neuroanaesthesiol Crit Care 2017;4:17-22. 
physiological and psychological responses to major surgeries ${ }^{[8]}$ which significantly affect the outcome. The key elements of the ERAS protocol include pre-operative counselling, pre-operative enteral and immune nutrition, avoidance of perioperative fasting and carbohydrate loading up to $2 \mathrm{~h}$ preoperatively, standardised anaesthetic and analgesic regimens, early institution of enteral nutrition (EN) and early mobilisation. In craniotomies, some concepts of ERAS may not be applicable and newer concepts such as role of scalp block and use of minimal access surgery (MAS), wherever applicable, are playing a pivotal role in enhanced recovery after craniotomies. ${ }^{[7]}$ The role of ERAS in major spine surgery ${ }^{[9]}$ is immense considering the wide variation in the length of stay, post-operative pain and functional recovery. Basically, the key ERAS component is thorough pre-operative, detailed and monitored intra- and post-operative care, as described in Figure 1.

\section{PREOPERATIVE}

\section{Counselling}

Educating patient may significantly impact patient approval and increase a patient's awareness of his/her surgical outcomes. Making the patients well learned of their long-term functional status builds self-confidence which can have a positive impact on their outcome. Studies have suggested interactive programmes focused on counselling and education which play a good deal of role in improving patient education and satisfaction. ${ }^{[10]}$

\section{Nutrition}

Poor pre-operative nutritional status is an attendant predictor of increased morbidity and length of stay in the post-operative period, whereas pre-operative EN improves outcomes. ${ }^{[11]}$ Recently, the focus has been shifted to the potential benefits of immunonutrition (IN). IN may enhance immune cell response, allowing for adaptation to systemic inflammation and oxidative

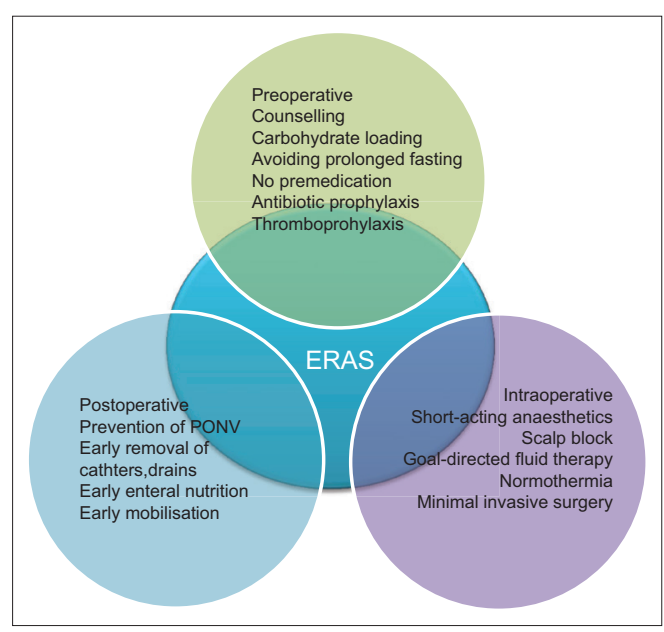

Figure 1: Key components of enhanced recovery after surgery stress. IN may be superior to EN for patients with cancer as it may enhance immune cell response, allowing more tolerance to oxidative stress. However, the generalizability of the same is disregarded as some of the trials were biased on this issue. ${ }^{[12]}$ A recent meta-analysis has shown the beneficial effects in terms of reduced length of hospital stay following arginine-enriched EN given for 5-10 days preoperatively and/or 7-10 days postoperatively. ${ }^{[13]}$

\section{Smoking and alcohol consumption}

Both smoking and alcohol consumption are known risk factors for increased morbidity and mortality postoperatively. Abstinence from alcohol for 1 month prior to elective surgery has been shown to reduce post-operative morbidity amongst alcohol abusers. ${ }^{[14]}$ Short-term smoking cessation of $<4$ weeks neither increases nor reduces the risk of perioperative respiratory complications. ${ }^{[15]}$ However, at least 4 weeks of abstinence from smoking reduces pulmonary complications, and abstinence of at least 3-4 weeks decreases wound-healing complications. ${ }^{[15]}$

\section{Pre-operative fasting and carbohydrate loading}

Preoperative oral carbohydrate loading up to $2 \mathrm{~h}$ in the pre-surgical period has been implicated to attenuate insulin resistance by activating the phosphatidylinositol 3 -kinase/protein kinase B signalling pathway. ${ }^{[16]}$ In addition, this leads to improved subjective feelings of hunger, thirst and post-operative fatigue compared with fasting. ${ }^{[16]}$ This practice does not increase the risk of aspiration and results in a shorter length of stay and an early return of bowel function in patients of colorectal surgery. ${ }^{[17]}$ Though the evidence level for such practice in neurosurgery is quite low, the recommendation grade is strong for such strategy. ${ }^{[7]}$

\section{Antithrombotic prophylaxis}

In the setting of craniotomy, patients are considered to be at a high risk of developing venous and arterial thrombosis which further compromises the quality of life. In this regard, antithrombotic prophylaxis is of greater value in terms of early discharge. Typically, in craniotomy population, the mechano-prophylaxis is generally preferred over pharmaco-prophylaxis because of the fear of bleeding complication which might pose a significant risk in a rigid cranium, especially in post-craniotomy patients. Mechano-prophylaxis includes the use of graduated compression stockings and intermittent pneumatic compression devices and has been found to be efficient in reducing the risk of venous thromboembolism (VTE). The major concern is addition of low molecular weight heparin, which in studies has been shown to decrease VTE, but it is also associated with an increase in major bleeding of borderline statistical 
significance. ${ }^{[18]}$ Pharmacologic prophylaxis should be tailored according to the individual risks involved. ${ }^{[19]}$

\section{Antimicrobial prophylaxis}

Neurosurgical patients exhibit a high risk of post-operative infection. A protocol-based perioperative antibiotic prophylaxis definitely reduces the surgical site infection but not the incidence of meningitis. ${ }^{[20]}$ This approach not only decreases surgical site infection but also tends to reduce extra-neurosurgical site infections. ${ }^{[21]}$ However, a recent meta-analysis demonstrates a significant reduction in meningitis incidence after antimicrobial prophylaxis. ${ }^{[22]}$ Mostly, the current practice is to administer cefazolin as the first-line choice for antibiotic prophylaxis in craniotomies within $60 \mathrm{~min}$ prior to skin incision. ${ }^{[23,24]}$ An exception to this recommendation is the prevalence of methicillin-resistant Staphylococcus aureus, for which a dose of vancomycin is generally recommended. ${ }^{[25,26]}$

\section{INTRAOPERATIVE}

\section{Scalp infiltrations and blocks}

Scalp infiltration and scalp block demonstrate reduced haemodynamic stress in terms of better perioperative control of haemodynamic, reduced opioids requirement with significantly low visual analogue scores postoperatively. ${ }^{[27]}$ Meta-analysis of scalp blocks has shown a significant mean reduction occurring at $1 \mathrm{~h}$ postoperatively. ${ }^{[28]}$ Although there is a paucity of randomised control trial (RCT) in suggesting the role of scalp blocks in post-craniotomy pain, still it can be a modality for reducing post-craniotomy pain which can have a significant impact on ERAS.

\section{Anaesthetic protocol}

A rapid recovery after anaesthesia for craniotomy allows prompt neurological assessment and diagnosis of intracranial complications at an early stage which can possibly lead to early hospital discharge. ${ }^{[29]}$ The impact of anaesthetic protocol on neurosurgical well-being after surgery is a topic of discussion for decades. Several studies comparing total intravenous anaesthetic versus inhalational anaesthetic (typically sevoflurane) have been carried out, but none of them concluded the benefit of one over other ${ }^{[30]}$ Nitrous oxide has been shown to increase cerebral metabolic rate, cerebral blood flow, intracranial pressure and post-operative nausea and vomiting (PONV) incidence, hence it is not widely used in neuroanaesthetic practice.

The role of magnesium sulphate, dexmedetomidine and lidocaine in perioperative medicine has been investigated as an adjunct to general anaesthesia and as potential modalities for acute post-operative pain control. Use of ketamine is associated with some undesirable side effects such as hallucinations, nausea and vomiting and blurred vision which make it unsuitable in the craniotomy population. Awake craniotomy is associated with good outcomes compared with surgery under general anaesthesia and can be considered as the standard of care for tumours in close proximity to the eloquent brain. ${ }^{[29]}$

\section{Analgesia}

In neurosurgical patients, the major task is to select analgesics for perioperative pain control, which have minimal effect on cognition and orientation. The anaesthesia protocol should also exclude longer acting opioids due to their undesirable side effects, such as sedation, miosis, nausea and vomiting, which may hinder the prompt recognition of incipient intracranial catastrophe. The respiratory depressant effects of opioids can increase the plasma carbon dioxide tension, which can ultimately lead to adverse intracranial haemodynamics. ${ }^{[31]}$ Though perioperative use of pregabalin attenuates anxiety and reduces post-operative pain scores and analgesic usage, ${ }^{[32]}$ its safety profile and adverse effects are always a concern in neurosurgical population. ${ }^{[33]}$ Non-steroidal anti-inflammatory agents are effective analgesics, but bleeding concerns limit the utilisation in craniotomy population. Hence, the appropriate selection of perioperative analgesics plays an important component of ERAS in craniotomy patients. In this particular setting, intravenous acetaminophen is more promising, but there are no studies to substantiate the effectiveness of this drug. In neurocritical care settings, a multinational survey found that the use of acetaminophen is the most common first-line analgesic and opioids, gabapentin to follow. ${ }^{[34]}$

\section{Fluids}

Consensus guidelines for perioperative fluid management in the fields of colorectal, ${ }^{[1-3]}$ bladder, ${ }^{[4]}$ pancreatic and gastrointestinal ${ }^{[5]}$ surgeries have been published in recent years. These guidelines emphasises the use of 'near zero' fluid balance. But to date, there is no consensus regarding the practice of perioperative fluid management in the neurosurgical scenario. The whole picture gets even more complicated with the use of diuretics and complications inherent to neurosurgical population. Nevertheless, prevention of negative fluid balance is imperative. Intra-operative use of goal-directed fluid therapy (GDT) has proven to be associated with decreased morbidity and lesser hospital stay, and when used in an ERAS setting, the length of stay in intensive care was reduced. ${ }^{[35]}$ The use of GDT in craniotomy setting as a component of ERAS is to be elucidated.

\section{Maintenance of core body temperature}

Maintenance of core body temperature is essential to anaesthetic management. It is well known that hypothermia prolongs the neuromuscular blockade and hence prolongs recovery from anaesthesia. ${ }^{[36]}$ 


\section{Minimal access surgery}

Early resumption of daily chores and improved self-perception make MAS a more attractive option from both the patients' and surgeons' perspective. Less post-operative pain primarily as a result of less tissue dissection is an additional benefit of MAS. Cosmetic satisfaction, ${ }^{[37]}$ less post-operative pain and early discharge make minimally invasive craniotomy and endoscopic approach as important components of ERAS, but to date, there are no RCTs depicting the quality of life following such procedures. ${ }^{[38]}$

\section{POST-OPERATIVE}

\section{Post-operative nausea and vomiting}

PONV is a distressing experience after recovery and attributed to various factors. The incidence of PONV in the post-craniotomy population is around $47 \%{ }^{[39]}$ Prompt control of PONV is necessary in the post-operative period as this may disturb the intracranial homeostasis. Serotonin receptor antagonism and dexamethasone in this regard are considered the suitable agents and are strongly recommended. Recently, a prospective trial showed similar effectiveness of aprepitant to ondansetron in controlling PONV in craniotomy population. ${ }^{[40]}$ There are studies suggesting the potential role of scopolamine combined with other existing therapies as a potent antiemetic. ${ }^{[41]}$ Another interesting non-pharmacological method is the use of transcutaneous electrical acupoint stimulation which has been shown to decrease the incidence of vomiting and nausea as well as post-operative pain in craniotomy individuals. ${ }^{[42]}$ The complementary use of acupoint stimulation for craniotomy has additional analgesic effects, reduces the onset of PONV and might have neuroprotective effects. ${ }^{[43]}$

\section{Post-operative nutrition}

Due to high levels of catabolism and hypermetabolism, these patients need adequate calories to sustain resting energy expenditure. In a study on the effect of early nutrition on mortality due to severe traumatic brain injury, the authors observed that for every $10 \mathrm{kcal} / \mathrm{kg}$, decrease in caloric intake was associated with a 30\%-40\% increase in mortality rates. ${ }^{[4]}$ A negative nitrogen balance is associated with an increased risk of hospital-acquired infections and a poorer outcome following aneurysmal subarachnoid patients. ${ }^{[45]}$ Most of the existing literatures suggest that early nutrition is beneficial in neurosurgical population and thus can lead to early recovery with good functional outcomes.

\section{Early ambulation}

Early ambulation prevents deep venous thrombosis and reduces the length of stay. ${ }^{[46]}$ Adequate control over pain, PONV and early nutritional support help achieve early mobilisation. The use of invasive lines and catheters should be minimised whenever possible in the post-craniotomy population, which also contributes to early mobilisation and discharge.

\section{FUTURE STRATEGIES}

A recent concept implicating ERAS in craniotomy called neurosurgery ERAS, value and safety (NERVS) is in the developmental stage, ${ }^{[47]}$ which has also been tested in microvascular decompression procedures for trigeminal neuralgia patients. ${ }^{[48]}$ Basically, NERVS consists of a multidisciplinary team, which has process mapped the way the ongoing healthcare being delivered throughout the entire episode of care. ${ }^{[47]}$

\section{CONCLUSION}

The application of ERAS has certainly changed the perioperative practice to have a smoother ride for the patients throughout the perioperative period of stress. ERAS is simply a careful coordinated multidisciplinary approach, adherence to which has better clinical outcome. The application of ERAS to craniotomy population has significant potential for better outcome. In patients undergoing craniotomy considerations such as IN, techniques for scalp blocks, non-opioid alternatives for pain control and improved outcomes with minimally invasive surgery are little different from the traditional ERAS concept. Adherence to ERAS for craniotomies may improve patient outcomes, accelerate functional recovery and decrease length of stay. However, prior testing an ERAS model for craniotomies is needed. The value and safety of such a strategy is to be tested before full implementation. More researches are needed in future to formulate a proper strategy for craniotomy patients to have a better perioperative outcome.

\section{Financial support and sponsorship} Nil.

\section{Conflicts of interest}

There are no conflicts of interest.

\section{REFERENCES}

1. Gustafsson UO, Scott MJ, Schwenk W, Demartines N, Roulin D, Francis N, et al. Guidelines for perioperative care in elective colonic surgery: Enhanced Recovery After Surgery $\left(\right.$ ERAS $^{\circledR}$ ) Society recommendations. World J Surg 2013;37:259-84.

2. Nelson G, Altman AD, Nick A, Meyer LA, Ramirez PT, Achtari C, et al. Guidelines for pre- and intra-operative care in gynecologic/oncology surgery: Enhanced Recovery After Surgery $\left(\right.$ ERAS $\left.^{\circledR}\right)$ Society recommendations - Part I. Gynecol Oncol 2016;140:313-22.

3. Nygren J, Thacker J, Carli F, Fearon KC, Norderval S, Lobo DN, et al. Guidelines for perioperative care in elective rectal/pelvic surgery: Enhanced Recovery After Surgery (ERAS ${ }^{\circledR}$ ) Society recommendations. World J Surg 2013;37:285-305. 
4. Cerantola Y, Valerio M, Persson B, Jichlinski P, Ljungqvist O, Hubner $\mathrm{M}$, et al. Guidelines for perioperative care after radical cystectomy for bladder cancer: Enhanced Recovery After Surgery $\left(\right.$ ERAS $\left.^{\circledR}\right)$ society recommendations. Clin Nutr 2013;32:879-87.

5. Mortensen K, Nilsson M, Slim K, Schäfer M, Mariette C, Braga $\mathrm{M}$, et al. Consensus guidelines for enhanced recovery after gastrectomy: Enhanced Recovery After Surgery (ERAS ${ }^{\circledR}$ ) Society recommendations. Br J Surg 2014;101:1209-29.

6. Kehlet $\mathrm{H}$. Multimodal approach to control postoperative pathophysiology and rehabilitation. $\mathrm{Br} \mathrm{J}$ Anaesth 1997;78:606-17.

7. Hagan KB, Bhavsar S, Raza SM, Arnold B, Arunkumar R, Dang A, et al. Enhanced recovery after surgery for oncological craniotomies. J Clin Neurosci 2016;24:10-6.

8. Fearon $\mathrm{KC}$, Ljungqvist $\mathrm{O}$, Von Meyenfeldt $\mathrm{M}$, Revhaug $\mathrm{A}$, Dejong $\mathrm{CH}$, Lassen $\mathrm{K}$, et al. Enhanced recovery after surgery: A consensus review of clinical care for patients undergoing colonic resection. Clin Nutr 2005;24:466-77.

9. Wainwright TW, Immins T, Middleton RG. Enhanced recovery after surgery (ERAS) and its applicability for major spine surgery. Best Pract Res Clin Anaesthesiol 2016;30:91-102.

10. Enzenhofer M, Bludau HB, Komm N, Wild B, Mueller K, Herzog W, et al. Improvement of the educational process by computer-based visualization of procedures: Randomized controlled trial. J Med Internet Res 2004;6:e16.

11. Bozzetti F, Gianotti L, Braga M, Di Carlo V, Mariani L. Postoperative complications in gastrointestinal cancer patients: The joint role of the nutritional status and the nutritional support. Clin Nutr 2007;26:698-709.

12. Burden S, Todd C, Hill J, Lal S. Pre-operative nutrition support in patients undergoing gastrointestinal surgery. Cochrane Database Syst Rev 2012;11:CD008879.

13. Vidal-Casariego A, Calleja-Fernández A, Villar-Taibo R, Kyriakos G, Ballesteros-PomarMD.Efficacy of arginine-enriched enteral formulas in the reduction of surgical complications in head and neck cancer: A systematic review and meta-analysis. Clin Nutr 2014;33:951-7.

14. Oppedal K, Møller AM, Pedersen B, Tønnesen H. Preoperative alcohol cessation prior to elective surgery. Cochrane Database Syst Rev 2012;7:CD008343.

15. Wong J, Lam DP, Abrishami A, Chan MT, Chung F. Short-term preoperative smoking cessation and postoperative complications: A systematic review and meta-analysis. Can J Anaesth 2012;59:268-79.

16. Wang ZG, Wang Q, Wang WJ, Qin HL. Randomized clinical trial to compare the effects of preoperative oral carbohydrate versus placebo on insulin resistance after colorectal surgery. Br J Surg 2010;97:317-27.

17. Jones C, Badger SA, Hannon R. The role of carbohydrate drinks in pre-operative nutrition for elective colorectal surgery. Ann R Coll Surg Engl 2011;93:504-7.

18. Salmaggi A, Simonetti G, Trevisan E, Beecher D, Carapella CM, DiMeco F, et al. Perioperative thromboprophylaxis in patients with craniotomy for brain tumours: A systematic review. J Neurooncol 2013;113:293-303.

19. Niemi T, Armstrong E. Thromboprophylactic management in the neurosurgical patient with high risk for both thrombosis and intracranial bleeding. Curr Opin Anaesthesiol 2010;23:558-63.

20. Korinek AM, Baugnon $\mathrm{T}$, Golmard JL, van Effenterre $\mathrm{R}$, Coriat P, Puybasset L. Risk factors for adult nosocomial meningitis after craniotomy: Role of antibiotic prophylaxis. Neurosurgery 2006;59:126-33.

21. Sharma MS, Vohra A, Thomas P, Kapil A, Suri A, Chandra PS, et al. Effect of risk-stratified, protocol-based perioperative chemoprophylaxis on nosocomial infection rates in a series of 31927 consecutive neurosurgical procedures (1994-2006) Neurosurgery 2009;64:1123-30.

22. Alotaibi AF, Hulou MM, Vestal M, Alkholifi F, Asgarzadeh M, Cote DJ, et al. The efficacy of antibacterial prophylaxis against the development of meningitis after craniotomy: A meta-analysis. World Neurosurg 2016;90:597-603.e1.

23. WHO. The Surgical Safety Checklist. Geneva: World Health Organisation; 2008. Available from: http://www.who.int/ patientsafety/safesurgery/tools resources/SSSL Manual finalJun08.pdf. [Last accessed on 2016 Nov 08].

24. Woods RK, Dellinger EP. Current guidelines for antibiotic prophylaxis of surgical wounds. Am Fam Physician 1998;57:2731-40.

25. Hammond CJ, Gill J, Peto TE, Cadoux-Hudson TA, Bowler IC. Investigation of prevalence of MRSA in referrals to neurosurgery: Implications for antibiotic prophylaxis. $\mathrm{Br} \mathrm{J}$ Neurosurg 2002;16:550-4.

26. Gemmell CG, Edwards DI, Fraise AP, Gould FK, Ridgway GL, Warren RE; Joint Working Party of the British Society for Joint Working Party of the British Society for Antimicrobial Chemotherapy, et al. Guidelines for the prophylaxis and treatment of methicillin-resistant Staphylococcus aureus (MRSA) infections in the UK. J Antimicrob Chemother 2006;57:589-608.

27. Hansen MS, Brennum J, Moltke FB, Dahl JB. Pain treatment after craniotomy: Where is the (procedure-specific) evidence? A qualitative systematic review. Eur J Anaesthesiol 2011;28:821-9.

28. Guilfoyle MR, Helmy A, Duane D, Hutchinson PJ. Regional scalp block for postcraniotomy analgesia: A systematic review and meta-analysis. Anesth Analg 2013;116:1093-102.

29. Gruenbaum SE, Meng L, Bilotta F. Recent trends in the anesthetic management of craniotomy for supratentorial tumor resection. Curr Opin Anaesthesiol 2016;29:552-7.

30. Prabhakar H, Singh GP, Mahajan C, Kapoor I, Kalaivani M, Anand V. Intravenous versus inhalational techniques for rapid emergence from anaesthesia in patients undergoing brain tumour surgery. Cochrane Database Syst Rev 2016;9:CD010467.

31. Cold GE, Felding M. Even small doses of morphine might provoke "luxury perfusion" in the postoperative period after craniotomy. Neurosurgery 1993;32:327.

32. Shimony N, Amit U, Minz B, Grossman R, Dany MA, Gonen L, et al. Perioperative pregabalin for reducing pain, analgesic consumption, and anxiety and enhancing sleep quality in elective neurosurgical patients: A prospective, randomized, double-blind, and controlled clinical study. J Neurosurg 2016;125:1513-22.

33. Salinsky M, Storzbach D, Munoz S. Cognitive effects of pregabalin in healthy volunteers: A double-blind, placebo-controlled trial. Neurology 2010;74:755-61.

34. Zeiler FA, AlSubaie F, Zeiler K, Bernard F, Skrobik Y. Analgesia in neurocritical care: An international survey and practice audit. Crit Care Med 2016;44:973-80.

35. Rollins KE, Lobo DN. Intraoperative goal-directed fluid therapy in elective major abdominal surgery: A meta-analysis of randomized controlled trials. Ann Surg 2016;263:465-76.

36. Lenhardt R, Marker E, Goll V, Tschernich H, Kurz A, Sessler DI, et al. Mild intraoperative hypothermia prolongs postanesthetic recovery. Anesthesiology 1997;87:1318-23.

37. Reisch R, Marcus HJ, Hugelshofer M, Koechlin NO, Stadie A, Kockro RA. Patients' cosmetic satisfaction, pain, and functional outcomes after supraorbital craniotomy through an eyebrow incision. J Neurosurg 2014;121:730-4.

38. Abdel Aziz KM, Bhatia S, Tantawy MH, Sekula R, Keller JT, Froelich S, et al. Minimally invasive transpalpebral "eyelid" approach to the anterior cranial base. Neurosurgery 
2011;69:ons195-206.

39. Latz B, Mordhorst C, Kerz T, Schmidt A, Schneider A, Wisser $\mathrm{G}$, et al. Postoperative nausea and vomiting in patients after craniotomy: Incidence and risk factors. J Neurosurg 2011;114:491-6.

40. Bergese SD, Puente EG, Antor MA, Viloria AL, Yildiz V, Kumar NA, et al. A prospective, randomized, double-blinded, double-dummy pilot study to assess the preemptive effect of triple therapy with aprepitant, dexamethasone, and promethazine versus ondansetron, dexamethasone and promethazine on reducing the incidence of postoperative nausea and vomiting experienced by patients undergoing craniotomy under general anesthesia. Front Med (Lausanne) 2016;3:29.

41. Bergese SD, Antor MA, Uribe AA, Yildiz V, Werner J. Triple therapy with scopolamine, ondansetron, and dexamethasone for prevention of postoperative nausea and vomiting in moderate to high-risk patients undergoing craniotomy under general anesthesia: A pilot study. Front Med (Lausanne) 2015;2:40.

42. An LX, Chen X, Ren XJ, Wu HF. Electro-acupuncture decreases postoperative pain and improves recovery in patients undergoing a supratentorial craniotomy. Am J Chin Med 2014;42:1099-109.

43. Asmussen S, Maybauer DM, Chen JD, Fraser JF, Toon MH, Przkora $\mathrm{R}$, et al. Effects of acupuncture in anesthesia for craniotomy: A meta-analysis. J Neurosurg Anesthesiol 2016. [Epub ahead of print].

44. Härtl R, Gerber LM, Ni Q, Ghajar J. Effect of early nutrition on deaths due to severe traumatic brain injury. J Neurosurg 2008;109:50-6.

45. Badjatia N, Monahan A, Carpenter A, Zimmerman J, Schmidt JM, Claassen J, et al. Inflammation, negative nitrogen balance, and outcome after aneurysmal subarachnoid hemorrhage. Neurology 2015;84:680-7.

46. Pashikanti L, Von Ah D. Impact of early mobilization protocol on the medical-surgical inpatient population: An integrated review of literature. Clin Nurse Spec 2012;26:87-94.

47. McLaughlin N, Rodstein J, Burke MA, Martin NA. Demystifying process mapping: A key step in neurosurgical quality improvement initiatives. Neurosurgery 2014;75:99-109.

48. McLaughlin N, Buxey F, Chaw K, Martin NA. Value-based neurosurgery: The example of microvascular decompression surgery. J Neurosurg 2014;120:462-72. 